\title{
CONDITIONS FOR THE COINCIDENCE OF THE LS AND AITKEN ESTIMATIONS OF THE HIGHER COEFFICIENT OF THE QUADRATIC REGRESSION MODEL
}

\author{
MARTA SAVKINA
}

Institute of Mathematics of NASU, Kyiv, Ukraine, E-mail: marta@imath.kiev.ua

\section{УМОВИ ЗБІГУ ОЦІНОК МНК ТА ЕЙТКЕНА СТАРШОГО КОЕФІЦІЕНТУ МОДЕЛІ КВАДРАТИЧНОЇ РЕГРЕСІЇ}

\author{
M. Ю. САBKIHA \\ Інститут математики НАНУ , Київ, Україна, E-mail: marta@imath.kiev.ua
}

\begin{abstract}
In the paper in the case of heteroscedastic independent deviations a regression model whose function has the form $f(x)=$ $a x^{2}+b x+c$, where $a, b$, and $c$ are unknown parameters, is studied. Approximate values (observations) of functions $f(x)$ are registered at equidistant points of a line segment. The theorem proved in the paper states that Aitken estimation of the higher coefficient of the quadratic model in the case of odd the number of observation points coincides with its estimation of LS iff values of the variances satisfy a certain system of nonlinear equations. Under these conditions, the Aitken and LS estimations of $b$ and $c$ will not coincide. The application of the theorem for some cases of a specific quantity of observation points and the same values of the variances at nodes symmetric about the point $\frac{1}{2}$ is considered. In all these cases it is obtained that the LS estimation will be coincide Aitken estimation if the variance in two points accepts arbitrary values, and at all others does certain values that are expressed through the values of variances in these two points.
\end{abstract}

KEYWORDS: least square method, regression model, Aitken estimation.

АнотАція. У роботі у випадку гетероскедастичних незалежних відхилень вивчається регресійна модель, функція якої має вигляд $f(x)=a x^{2}+b x+c$, де $a, b$ та $c-$ невідомі параметри. Наближені значення (спостереження) функції $f(x)$ реєструються у рівновіддалених точках відрізку $[0,1]$. Теорема, яку доведено в роботі, стверджує, що оцінка Ейткена старшого коефіціенту квадратичної моделі у випадку непарної кількості точок спостереження збігається з його оцінкою МНК тоді і тільки тоді, коли значення дисперсій відхилень задовільняють певній системі нелінійних рівнянь. При цих умовах оцінки Ейткена та МНК параметрів $b$ та 
$c$ не будуть збігатися. Розглянуто застосування теореми для деяких випадків конкретної кількості точок спостереження та однакових значеннях дисперсій у вузлах, симетричних відносно точки $\frac{1}{2}$. В усіх цих випадках отримано, що оцінка МНК буде збігатися 3 оцінкою Ейткена, якщо дисперсія в двох точках приймає довільні значення, а в усіх інших - певні значення, які виражаються через значення дисперсій в цих двох точках.

Ключові СловА: метод найменших квадратів, регресійна модель, оцінка Ейткена.

\section{ВСТУП}

В класичному регресійному аналізі в якості випадкових відхилень беруть послідовність незалежних у сукупності випадкові величини з однаковою дисперсією. Така умова досить обмежувальна, на практиці вона часто не виконується. Фактично припущення класичної регресії означає, що невраховані фактори діють випадково від експеримента до експерименту, а також розкид таких факторів сталий. В зв'язку з цим була розвинута теорія для дослідження регресійної моделі, в якій відхилення корельовані, або принаймні, гетероскедастичні. Проте в такому випадку оцінка звичайного методу найменших квадратів (MHK) невідомих параметрів моделі хоч і буде незміщеною та спроможною, вже не буде ефективною [2]. Для того, щоб отримати оцінку, яка має мінімальну дисперсію в класі незміщених лінійних оцінок, треба видозмінити МНК. Оцінка з мінімальною дисперсію називається марковською або оцінкою Ейткена; в формулу для неї входить коваріаційна матриця відхилень, яка часто буває невідомою. А оцінка МНК завжди обчислювана.

Отже, порівняння цих оцінок, встановлення зв'язку між ними, а також знаходження випадків, коли вони збігаються, має важливе значення. Якщо вони відрізняються одна від одної, досліджується задача, яку похибку ми допускаємо, коли використовуємо оцінку МНК замість оцінки Ейткена. В якості міри ефективності беруть відношення визначників коваріаційних матриць цих оцінок.

В [1] у випадку моделі, лінійної по параметрам, доведено декілька теорем, які дають необхідні та достатні умови на матрицю плану для збігу оцінки МНК та оцінки Ейткена. Ватсон [7-8], Хеннан [9], Зискинд [4], Магнес та Макгир [5] досдіджували ефективність, Гренандер [3] та Розенблат [6] - асимптотичну ефективність оцінки МНК.

В статті [10] вивчається властивість рівності оцінок параметрів лінійної множинної регресійній моделі, отриманих методом найменших квадратів та узагальненим МНК при значно відмінних похибках у вихідних данних. В статті [11] у випадку гетероскедастичних незалежних відхилень вивчається регресійна модель, функція якої має вигляд $f(x)=a x+b$. Знайдено умови на дисперсії відхилень, при яких оцінка Ейткена збігається з оцінкою МНК окремо для кожного невідомого параметру моделі. При цих умовах оцінки Ейткена та МНК іншого параметру не будуть збігатися. 
1. ОЦІНКА МНК ТА ОЦІНКА ЕЙТКЕНА СТАРШОГО КОЕФІЦІЕНТУ КВАДРАТИЧНОЇ РЕГРЕСІЙНОЇ МОДЕЛІ У ВИПАДКУ ГЕТЕРОСКЕДАСТИЧНИХ НЕЗАЛЕЖНИХ ВІДХИЛЕНЬ

Розглянемо модель регресії

$$
y_{i}=a t_{i}^{2}+b t_{i}+c+\epsilon_{i}, \quad i=0,1, \ldots, n,
$$

де $a, b$ і $c$ - невідомі параметри, які підлягають оцінюванню, $\epsilon_{0}, \ldots, \epsilon_{n}-$ незалежні у сукупності випадкові величини з $E \epsilon_{i}=0$ та $D \epsilon_{i}=\lambda_{i} \sigma^{2}, \lambda_{i}>0$, $i=0,1, \ldots, n$.

В [2] знайдено формули для оцінки МНК та оцінки Ейткена невідомих параметрів моделі регресії загального вигляду, лінійної по параметрам. 3 цих формул у випадку $t_{i}=\frac{i}{n}, i=0,1, \ldots, n$, маємо такі оцінки MНК та Ейткена параметра $a$ моделі (1):

$$
\begin{gathered}
\hat{a}_{M N K}=\frac{180 n}{(n+1)(n+2)(n-1)(n+3)} \sum_{i=0}^{n} z(i) y_{i}, \\
\hat{a}_{A I T}=n^{2} \Delta_{n}^{-1} \sum_{i=0}^{n} \lambda_{i}\left(\sum_{l=0, j>l}^{n} \lambda_{l} \lambda_{j}(l-j)^{2}(i-l)(i-j)\right) y_{i},
\end{gathered}
$$

де

$$
\begin{gathered}
z(i)=\frac{n(n+2)}{12}-\left(i-\frac{n}{2}\right)^{2} \\
\Delta_{n}=\sum_{i=0}^{n} i^{2} \lambda_{i}\left(\sum_{l=0, j>l}^{n} \lambda_{l} \lambda_{j}(l-j)^{2}(i-l)(i-j)\right) .
\end{gathered}
$$

2. УМОВИ НА ДИСПЕРСІЇ ВІДХИЛЕНЬ ДЛЯ ЗБІГУ ОЦННОК МНК ТА ЕЙТКЕНА СТАРШОГО КОЕФІЦІЕНТУ КВАДРАТИЧНОЇ РЕГРЕСІЙНОЇ МОДЕЛІ

Має місце

Теорема 1. Оиінка Ейткена та оцінка МНК параметра а в моделі (1) у випадку парного п збігаються тоді $i$ тільки тодi, коли $\lambda_{i}, i=0,1, \ldots, n, e$ розв'язком наступної системи рівнянь

$$
\begin{gathered}
\lambda_{i} \sum_{l=0, j>l}^{n} \lambda_{l} \lambda_{j}(l-j)^{2}(i-l)(i-j)= \\
=\frac{12 \lambda_{\frac{n}{2}} z(i)}{n(n+2)} \sum_{l=0, j>l}^{n} \lambda_{l} \lambda_{j}(l-j)^{2}\left(\frac{n}{2}-l\right)\left(\frac{n}{2}-j\right), \\
i=0,1, \ldots, \frac{n}{2}-1, \frac{n}{2}+1, \ldots, n .
\end{gathered}
$$

Доведення. Необхідність. Нехай

$$
\hat{a}_{A I T}=\hat{a}_{M N K} .
$$


3 (4) отримуємо рівність

$$
\sum_{i=0}^{n} \alpha_{i} y_{i}=0
$$

де

$$
\begin{aligned}
\alpha_{i}=\frac{\lambda_{i} n(n+1)}{(n+2)(n-1)(n+3)} \times & 180 \\
\times & \times \sum_{l=0, j>l}^{n} \lambda_{l} \lambda_{j}(l-j)^{2}(i-l)(i-j)-z(i) \Delta_{n}, \quad i=0,1, \ldots, n .
\end{aligned}
$$

Для того, щоб (5) виконувалось для будь-яких $y_{i}, i=0,1, \ldots, n$, необхідно і достатньо, щоб

$$
\alpha_{i}=0, i=0,1, \ldots, n .
$$

$\mathrm{У}$ випадку парного $n$ з (6) при $i=\frac{n}{2}$ маємо рівняння

$$
\begin{gathered}
\lambda_{\frac{n}{2}} \sum_{l=0, j>l}^{n} \lambda_{l} \lambda_{j}(l-j)^{2}\left(\frac{n}{2}-l\right)\left(\frac{n}{2}-j\right)= \\
=-\frac{15 \Delta_{n}}{(n+1)(n-1)(n+3)} .
\end{gathered}
$$

3 усіх інших рівнянь системи (6) отримуємо

$$
\begin{gathered}
\lambda_{i} z^{-1}(i) \sum_{l=0, j>l}^{n} \lambda_{l} \lambda_{j}(l-j)^{2}(i-l)(i-j)= \\
=\frac{12}{n(n+2)} \cdot \frac{15 \Delta_{n}}{(n+1)(n-1)(n+3)}, i=0,1, \ldots, \frac{n}{2}-1, \frac{n}{2}+1, \ldots, n,
\end{gathered}
$$

якщо $z(i) \neq 0$, або

$$
\sum_{l=0, j>l}^{n} \lambda_{l} \lambda_{j}(l-j)^{2}(i-l)(i-j)=0, \text { якщо } z(i)=0 .
$$

Далі, бачимо, що якщо $z(i) \neq 0$, то з $i$-го рівняння системи (8) та рівняння (7) випливає $i$-е рівняння системи (3). А якщо $z(i)=0$, то $i$-е рівняння системи (8) збігається з $i$-м рівняння системи (3).

Неважко помітити, що рівняння (7) є лінійною комбінацією рівнянь (8), тобто система (8) є еквівалентною системі рівнянь (3).

Таким чином, з умови (4) випливає (3).

Достатність. Нехай $\lambda_{i}^{*}, i=0,1, \ldots, n, \in$ розв'язком системи (3). Оскільки системи (8) та (3) еквівалентні, а рівняння (7) є лінійною комбінацією рівнянь (8), то $\lambda_{i}^{*}, i=0,1, \ldots, n, \in$ розв'язком системи (8) та рівняння (7). Перепишемо отримані з (7) та (8) тотожності наступним чином

$$
\Delta_{n}^{-1} \lambda_{\frac{n}{2}}^{*} \sum_{l=0, j>l}^{n} \lambda_{l}^{*} \lambda_{j}^{*}(l-j)^{2}\left(\frac{n}{2}-l\right)\left(\frac{n}{2}-j\right)=
$$




$$
\begin{gathered}
=\frac{12}{n(n+2)} \cdot \frac{15 z\left(\frac{n}{2}\right)}{(n+1)(n-1)(n+3)}, \\
\Delta_{n}^{-1} \lambda_{i}^{*} \sum_{l=0, j>l}^{n} \lambda_{l}^{*} \lambda_{j}^{*}(l-j)^{2}(i-l)(i-j)= \\
=\frac{12}{n(n+2)} \cdot \frac{15 z(i)}{(n+1)(n-1)(n+3)}, i=0,1, \ldots, \frac{n}{2}-1, \frac{n}{2}+1, \ldots, n .
\end{gathered}
$$

Далі, підставимо $\lambda_{i}^{*}, i=0,1, \ldots, n$, в формулу (2). Враховуючи тотожності $\left(7^{\prime}\right)$ та $\left(8^{\prime}\right)$, отримуємо

$$
\begin{gathered}
\hat{a}_{A I T}=\Delta_{n}^{-1} \sum_{i=0}^{n} \lambda_{i}^{*}\left(\sum_{l=0, j>l}^{n} \lambda_{l}^{*} \lambda_{j}^{*}(l-j)^{2}(i-l)(i-j)\right) y_{i}= \\
=\frac{180 n}{(n+1)(n+2)(n-1)(n+3)} \sum_{i=0}^{n} z(i) y_{i}=\hat{a}_{M N K},
\end{gathered}
$$

тобто маємо (4).

Теорему доведено.

Таким чином, для того, щоб знайти, при яких $\lambda_{i}, i=0,1, \ldots, n$, оцінки MHK та Ейткена параметру $a$ будуть збігатися, треба розв'язати систему рівнянь (3) відносно $\lambda_{0}, \lambda_{1}, \ldots, \lambda_{n}$.

3. ЗАСТОСУВАННЯ ТЕОРЕМИ ДЛЯ ДЕЯКИХ КОНКРЕТНИХ $n$ ТА ОДНАКОВИХ ЗНАЧЕННЯХ ДИСПЕРСІЙ У ТОЧКАХ СПОСТЕРЕЖЕННЯ, СИМЕТРИЧНИХ ВІДНОСНО ТОЧКИ $\frac{1}{2}$.

Розглянемо застосування теореми 1 для $n=4, n=6, n=8$ у випадку, коли

Має місце

$$
\lambda_{0}=\lambda_{n}, \quad \lambda_{1}=\lambda_{n-1}, \ldots, \lambda_{\frac{n}{2}-1}=\lambda_{\frac{n}{2}+1} .
$$

Наслідок 1. Якщо в моделі (1) умова $\lambda_{j}=\lambda, j=0,1, \ldots, n$, не виконуеться, то оцінка Ейткена та очінка МНК параметра а у випадку $n=4 \mathrm{ma}$ $\lambda_{i}=\lambda_{4-i}, i=0,1$ збігаються тоді і тільки тоді, коли $\lambda_{1}, \lambda_{2}-$ будъ-які, aлe $3 \lambda_{1}-2 \lambda_{2}>0, a$

$$
\lambda_{0}=\frac{\lambda_{1} \lambda_{2}}{3 \lambda_{1}-2 \lambda_{2}} .
$$

Доведення. У випадку $n=4$ система рівнянь (3) прийме вигляд

$$
\begin{aligned}
& \lambda_{0}\left(2 \lambda_{1} \lambda_{2}+12 \lambda_{1} \lambda_{3}+36 \lambda_{1} \lambda_{4}+6 \lambda_{2} \lambda_{3}+32 \lambda_{2} \lambda_{4}+12 \lambda_{3} \lambda_{4}\right)= \\
= & -\lambda_{2}\left(2 \lambda_{1} \lambda_{0}-18 \lambda_{0} \lambda_{3}-64 \lambda_{0} \lambda_{4}-4 \lambda_{1} \lambda_{3}-18 \lambda_{1} \lambda_{4}+2 \lambda_{3} \lambda_{4}\right) ; \\
& \lambda_{1}\left(-4 \lambda_{0} \lambda_{2}-18 \lambda_{0} \lambda_{3}-48 \lambda_{0} \lambda_{4}+2 \lambda_{2} \lambda_{3}+12 \lambda_{2} \lambda_{4}+6 \lambda_{3} \lambda_{4}\right)= \\
= & -\frac{1}{2} \lambda_{2}\left(2 \lambda_{1} \lambda_{0}-18 \lambda_{0} \lambda_{3}-64 \lambda_{0} \lambda_{4}-4 \lambda_{1} \lambda_{3}-18 \lambda_{1} \lambda_{4}+2 \lambda_{3} \lambda_{4}\right) ; \\
& \lambda_{3}\left(6 \lambda_{0} \lambda_{1}+12 \lambda_{0} \lambda_{2}-48 \lambda_{1} \lambda_{4}+2 \lambda_{1} \lambda_{2}-18 \lambda_{2} \lambda_{4}-4 \lambda_{3} \lambda_{4}\right)=
\end{aligned}
$$




$$
\begin{gathered}
=-\frac{1}{2} \lambda_{2}\left(2 \lambda_{1} \lambda_{0}-18 \lambda_{0} \lambda_{3}-64 \lambda_{0} \lambda_{4}-4 \lambda_{1} \lambda_{3}-18 \lambda_{1} \lambda_{4}+2 \lambda_{3} \lambda_{4}\right) ; \\
\lambda_{4}\left(12 \lambda_{0} \lambda_{1}+32 \lambda_{0} \lambda_{2}+36 \lambda_{0} \lambda_{3}+6 \lambda_{1} \lambda_{2}+12 \lambda_{1} \lambda_{3}+2 \lambda_{2} \lambda_{3}\right)= \\
=-\lambda_{2}\left(2 \lambda_{1} \lambda_{0}-18 \lambda_{0} \lambda_{3}-64 \lambda_{0} \lambda_{4}-4 \lambda_{1} \lambda_{3}-18 \lambda_{1} \lambda_{4}+2 \lambda_{3} \lambda_{4}\right) .
\end{gathered}
$$

Якщо $\lambda_{0}=\lambda_{4}, \lambda_{1}=\lambda_{3}, 3$ цих рівнянь маємо

$$
\begin{gathered}
\lambda_{0}\left(2 \lambda_{1} \lambda_{2}+12 \lambda_{1} \lambda_{0}+8 \lambda_{2} \lambda_{0}+3 \lambda_{1}^{2}\right)=\lambda_{2}\left(8 \lambda_{1} \lambda_{0}+16 \lambda_{0}^{2}+\lambda_{1}^{2}\right) ; \\
\lambda_{1}\left(4 \lambda_{0} \lambda_{2}-6 \lambda_{1} \lambda_{0}-24 \lambda_{0}^{2}+\lambda_{1} \lambda_{2}\right)=-\lambda_{2}\left(8 \lambda_{1} \lambda_{0}+16 \lambda_{0}^{2}+\lambda_{1}^{2}\right) .
\end{gathered}
$$

3 обох рівнянь (10) та (11) отримуємо

$$
\left(\left(3 \lambda_{1}-2 \lambda_{2}\right) \lambda_{0}-\lambda_{1} \lambda_{2}\right)\left(4 \lambda_{0}+\lambda_{1}\right)=0 .
$$

Оскільки $\lambda_{i}>0, i=0,1, \ldots, n$, то з рівняння (12) випливає (9) при умові, що $\lambda_{1}, \lambda_{2}-$ будь-які, але $3 \lambda_{1}-2 \lambda_{2}>0$.

Наслідок 2. Якщо в моделі (1) умова $\lambda_{j}=\lambda, j=0,1, \ldots, n$, не виконуеться, то очінка Ейткена та оцінка МНК параметра а у випадку $n=6$ mа $\lambda_{i}=\lambda_{6-i}, i=0,1,2$ збігаються тоді $i$ тільки тодi, коли $\lambda_{1}, \lambda_{2}-$ будъ-які, але $\lambda_{1} \neq \lambda_{2}, a$

$$
\lambda_{0}=\lambda_{3}=\lambda_{2} .
$$

Доведення. У випадку, коли $\lambda_{0}=\lambda_{6}, \lambda_{1}=\lambda_{5}, \lambda_{2}=\lambda_{4}$ система рівнянь (3) прийме вигляд

$$
\begin{gathered}
\lambda_{0}^{2}\left(180 \lambda_{1}+288 \lambda_{2}+162 \lambda_{3}\right)+ \\
+\lambda_{0}\left(80 \lambda_{1}^{2}+32 \lambda_{2}^{2}+148 \lambda_{1} \lambda_{2}+\lambda_{3}\left(18 \lambda_{2}+72 \lambda_{1}\right)\right)= \\
=-1,25 \lambda_{3}\left(-324 \lambda_{0}^{2}+\lambda_{0}\left(-288 \lambda_{1}-72 \lambda_{2}\right)-\right. \\
\left.-\left(64 \lambda_{1}^{2}+4 \lambda_{2}^{2}+32 \lambda_{1} \lambda_{2}\right)\right)
\end{gathered}
$$

у випадку $n=6$ маємо $z(1)=0$, тому

$$
\begin{gathered}
\lambda_{1}^{2}\left(-80 \lambda_{0}+48 \lambda_{2}+32 \lambda_{3}\right)+ \\
+\lambda_{1}\left(-180 \lambda_{0}^{2}+12 \lambda_{2}^{2}+88 \lambda_{0} \lambda_{2}+\lambda_{3}\left(72 \lambda_{0}+8 \lambda_{2}\right)\right)=0 ; \\
\lambda_{2}^{2}\left(-12 \lambda_{1}-32 \lambda_{0}+2 \lambda_{3}\right)+ \\
+\lambda_{2}\left(-48 \lambda_{1}^{2}-288 \lambda_{0}^{2}-236 \lambda_{0} \lambda_{1}+\lambda_{3}\left(18 \lambda_{0}+8 \lambda_{1}\right)\right)= \\
=0,75 \lambda_{3}\left(-4 \lambda_{2}^{2}+\lambda_{2}\left(-32 \lambda_{1}-72 \lambda_{0}\right)+\right. \\
\left.+\left(-64 \lambda_{1}^{2}-324 \lambda_{0}^{2}-288 \lambda_{1} \lambda_{0}\right)\right) .
\end{gathered}
$$

3 рівнянь (14), (15), (16) відповідно отримуємо

$$
\begin{gathered}
\left(\left(27 \lambda_{3}-20 \lambda_{1}-32 \lambda_{2}\right) \lambda_{0}+5\left(4 \lambda_{1}+\lambda_{2}\right) \lambda_{3}\right)\left(9 \lambda_{0}+4 \lambda_{1}+\lambda_{2}\right)=0 \\
\left(2 \lambda_{3}+3 \lambda_{2}-5 \lambda_{0}\right)\left(9 \lambda_{0}+4 \lambda_{1}+\lambda_{2}\right)=0 \\
\left(\left(5 \lambda_{3}-12 \lambda_{1}-32 \lambda_{0}\right) \lambda_{2}+3\left(9 \lambda_{0}+4 \lambda_{1}\right) \lambda_{3}\right)\left(9 \lambda_{0}+4 \lambda_{1}+\lambda_{2}\right)=0 .
\end{gathered}
$$

3 рівняння $\left(15^{\prime}\right)$ маємо

$$
\lambda_{0}=\frac{2 \lambda_{3}+3 \lambda_{2}}{5} .
$$


Підставляємо $(17)$ в $\left(14^{\prime}\right)$ та $\left(16^{\prime}\right)$, отримуємо відповідно $\lambda_{2}=\lambda_{3}$ та $\lambda_{2}=\lambda_{0}$, тобто маємо (13).

Лема 1. Якщо $\lambda_{2}>\lambda_{3}, \lambda_{3}>0$, то кубічне рівняння

$$
f(x)=0,
$$

$\partial e$

$$
\begin{gathered}
f(x)=360\left(-8,5 \lambda_{2}+\lambda_{3}\right) x^{3}+ \\
+\left(-2896,8 \lambda_{2}^{2}-437,4 \lambda_{2} \lambda_{3}+409,2 \lambda_{3}^{2}\right) x^{2}+ \\
+\left(2040 \lambda_{2}^{3}+8130 \lambda_{2}^{2} \lambda_{3}+6465 \lambda_{2} \lambda_{3}^{2}-480 \lambda_{3}^{3}\right) x- \\
-1800 \lambda_{2} \lambda_{3}\left(\lambda_{2}^{2}+2,85 \lambda_{2} \lambda_{3}+2 \lambda_{3}^{2}\right),
\end{gathered}
$$

мае принаймні один додатній корінь $x^{*}$, причому $x^{*}<\lambda_{3}$.

Доведення. Справді,

$$
f(0)=-1800 \lambda_{2} \lambda_{3}\left(\lambda_{2}^{2}+2,85 \lambda_{2} \lambda_{3}+2 \lambda_{3}^{2}\right)<0,
$$

a

$$
f\left(\lambda_{3}\right)=240\left(\lambda_{2}-\lambda_{3}\right)\left(\lambda_{2}-0,595 \lambda_{3}\right)\left(\lambda_{2}+2,025 \lambda_{3}\right)>0 .
$$

Таким чином, існуе $x^{*}, 0<x^{*}<\lambda_{3}$, такий що $f\left(x^{*}\right)=0$.

Наслідок 3. Якщо в моделі (1) у випадку $n=8$ mа $\lambda_{i}=\lambda_{8-i}, i=0,1,2,3$, $\lambda_{2}, \lambda_{3}-$ будъ-які, але $\lambda_{2}>\lambda_{3}, \lambda_{4}=\lambda_{4}^{*}, \lambda_{0}=\lambda_{0}^{*}$, ma $\lambda_{1}=\lambda_{1}^{*}$, де $\lambda_{4}^{*}-$ найменший додатній корінь рівняння (18),

$$
\begin{aligned}
& \lambda_{0}^{*}=\frac{1,4 \lambda_{4}^{*}\left(4 \lambda_{2}+\lambda_{3}\right)}{12 \lambda_{2}+15 \lambda_{3}-20 \lambda_{4}^{*}}, \\
& \lambda_{1}^{*}=\frac{1,4 \lambda_{4}^{*}\left(4 \lambda_{2}+\lambda_{3}\right)}{20 \lambda_{2}+32 \lambda_{3}-45 \lambda_{4}^{*}} .
\end{aligned}
$$

то оцінка Ейткена та оцінка МНК параметра а збігаютвся.

Доведення. У випадку, коли $\lambda_{0}=\lambda_{8}, \lambda_{1}=\lambda_{7}, \lambda_{2}=\lambda_{6}, \lambda_{3}=\lambda_{5}$ система рівнянь (3) прийме вигляд

$$
\begin{gathered}
64 \lambda_{0}^{2}\left(7 \lambda_{1}+12 \lambda_{2}+15 \lambda_{3}+8 \lambda_{4}\right)+ \\
+4 \lambda_{0}\left(8 \lambda_{4}\left(9 \lambda_{1}+4 \lambda_{2}+\lambda_{3}\right)+\right. \\
\left.+\left(\lambda_{3}+4 \lambda_{2}+9 \lambda_{1}\right)\left(15 \lambda_{3}+12 \lambda_{2}+7 \lambda_{1}\right)\right)= \\
=1,4 \lambda_{4}\left(1024 \lambda_{0}^{2}+128 \lambda_{0}\left(9 \lambda_{1}+4 \lambda_{2}+\lambda_{3}\right)+4\left(9 \lambda_{1}+4 \lambda_{2}+\lambda_{3}\right)^{2}\right) \\
18 \lambda_{1}^{2}\left(-14 \lambda_{0}+10 \lambda_{2}+16 \lambda_{3}+9 \lambda_{4}\right)+ \\
+2 \lambda_{1}\left(9 \lambda_{4}\left(16 \lambda_{0}+4 \lambda_{2}+\lambda_{3}\right)+\right. \\
\left.+2\left(16 \lambda_{0}+4 \lambda_{2}+\lambda_{3}\right)\left(8 \lambda_{3}+5 \lambda_{2}-7 \lambda_{0}\right)\right)= \\
=0,35 \lambda_{4}\left(324 \lambda_{1}^{2}+72 \lambda_{1}\left(16 \lambda_{0}+4 \lambda_{2}+\lambda_{3}\right)+4\left(16 \lambda_{0}+4 \lambda_{2}+\lambda_{3}\right)^{2}\right) ; \\
16 \lambda_{2}^{2}\left(-12 \lambda_{0}-5 \lambda_{1}+3 \lambda_{3}+2 \lambda_{4}\right)+ \\
+4 \lambda_{2}\left(2 \lambda_{4}\left(16 \lambda_{0}+9 \lambda_{1}+\lambda_{3}\right)+\right. \\
\left.+\left(16 \lambda_{0}+9 \lambda_{1}+\lambda_{3}\right)\left(3 \lambda_{3}-12 \lambda_{0}-5 \lambda_{1}\right)\right)= \\
=0,4 \lambda_{4}\left(64 \lambda_{2}^{2}+32 \lambda_{2}\left(16 \lambda_{0}+9 \lambda_{1}+\lambda_{3}\right)+4\left(16 \lambda_{0}+9 \lambda_{1}+\lambda_{3}\right)^{2}\right)
\end{gathered}
$$




$$
\begin{gathered}
\lambda_{3}^{2}\left(-60 \lambda_{0}-32 \lambda_{1}-12 \lambda_{2}+2 \lambda_{4}\right)+ \\
+\lambda_{3}\left(2 \lambda_{4}\left(16 \lambda_{0}+9 \lambda_{1}+4 \lambda_{2}\right)-\right. \\
\left.-\left(16 \lambda_{0}+9 \lambda_{1}+4 \lambda_{2}\right)\left(60 \lambda_{0}+32 \lambda_{1}+12 \lambda_{2}\right)\right)= \\
=0,85 \lambda_{4}\left(-4 \lambda_{3}^{2}-8 \lambda_{3}\left(16 \lambda_{0}+9 \lambda_{1}+4 \lambda_{2}\right)-4\left(16 \lambda_{0}+9 \lambda_{1}+4 \lambda_{2}\right)^{2}\right) ;
\end{gathered}
$$

3 рівнянь (21)-(24) відповідно отримуємо

$$
\begin{aligned}
& \left(\lambda_{0}\left(7 \lambda_{1}+12 \lambda_{2}+15 \lambda_{3}-14,4 \lambda_{4}\right)-1,4 \lambda_{4}\left(9 \lambda_{1}+4 \lambda_{2}+\lambda_{3}\right)\right) \sum_{i=0}^{3}(4-i)^{2}=0 \\
& \left(\lambda_{1}\left(28 \lambda_{0}-20 \lambda_{2}-32 \lambda_{3}-5,4 \lambda_{4}\right)+1,4 \lambda_{4}\left(16 \lambda_{0}+4 \lambda_{2}+\lambda_{3}\right)\right) \sum_{i=0}^{3}(4-i)^{2}=0 \\
& \left(\lambda_{2}\left(30 \lambda_{0}+12,5 \lambda_{1}-7,5 \lambda_{3}-9 \lambda_{4}\right)-\lambda_{4}\left(16 \lambda_{0}+9 \lambda_{1}+\lambda_{3}\right)\right) \sum_{i=0}^{3}(4-i)^{2}=0 \\
& \left(\lambda_{3}\left(60 \lambda_{0}+32 \lambda_{1}+12 \lambda_{2}-5,4 \lambda_{4}\right)-3,4 \lambda_{4}\left(16 \lambda_{0}+9 \lambda_{1}+4 \lambda_{2}\right)\right) \sum_{i=0}^{3}(4-i)^{2}=0 .
\end{aligned}
$$

$3\left(21^{\prime}\right)$ та $\left(22^{\prime}\right)$ маємо

та

$$
\lambda_{0}=\frac{1,4 \lambda_{4}\left(9 \lambda_{1}+4 \lambda_{2}+\lambda_{3}\right)}{7 \lambda_{1}+12 \lambda_{2}+15 \lambda_{3}-14,4 \lambda_{4}}
$$

$$
\lambda_{1}=\frac{1,4 \lambda_{4}\left(16 \lambda_{0}+4 \lambda_{2}+\lambda_{3}\right)}{28 \lambda_{0}-20 \lambda_{2}-32 \lambda_{3}-5,4 \lambda_{4}}
$$

відповідно. Підставимо $\left(21^{\prime \prime}\right)$ в $\left(22^{\prime \prime}\right)$, отримуємо

$$
\lambda_{1}=\frac{1,4 \lambda_{4}\left(4 \lambda_{2}+\lambda_{3}\right)}{20 \lambda_{2}+32 \lambda_{3}-45 \lambda_{4}} .
$$

Далі, підставимо (24) в (21"), отримуємо

$$
\lambda_{0}=\frac{1,4 \lambda_{4}\left(4 \lambda_{2}+\lambda_{3}\right)}{12 \lambda_{2}+15 \lambda_{3}-20 \lambda_{4}} .
$$

$3\left(23^{\prime}\right)$ та $\left(24^{\prime}\right)$ маємо відповідно

$$
\lambda_{2}\left(30 \lambda_{0}+12,5 \lambda_{1}-7,5 \lambda_{3}-9 \lambda_{4}\right)-\lambda_{4}\left(16 \lambda_{0}+9 \lambda_{1}+\lambda_{3}\right)=0
$$

та

$$
\lambda_{3}\left(60 \lambda_{0}+32 \lambda_{1}+12 \lambda_{2}-5,4 \lambda_{4}\right)-3,4 \lambda_{4}\left(16 \lambda_{0}+9 \lambda_{1}+4 \lambda_{2}\right) .
$$

Підставимо $\left(19^{\prime}\right)$ та $\left(20^{\prime}\right)$ в $\left(23^{\prime \prime}\right)$ та $\left(24^{\prime \prime}\right)$, отримуємо в обох випадках однакове кубічне рівняння (23) відносно $\lambda_{4}$.

Якщо $\lambda_{2}>\lambda_{3}$, в лемі 1 доведено, шо рівняння (18) має принаймні один додатній корінь $x^{*}$, причому $x^{*}<\lambda_{3}$. Позначимо найменший додатній корінь рівняння (18) через $\lambda_{4}^{*}$. Так як $\lambda_{4}^{*}<\lambda_{3}$, то легко бачити, що $\lambda_{0}^{*}$ та $\lambda_{1}^{*}$, отримані за формулами (19) та (20), будут також додатними. 


\section{4. Висновки}

У статті у випадку гетероскедастичних незалежних відхилень вивчено регресійну модель, функція якої має вигляд

$$
f(x)=a x^{2}+b x+c,
$$

де $a, b$ та $c$ - невідомі параметри. Наближені значення (спостереження) функції $f(x)$ реєструються у рівновіддалених точках відрізку $[0,1]$. Теорема 1 стверджує, що оцінка Ейткена старшого коефіціенту квадратичної моделі у випадку непарної кількості точок спостереження збігається з його оцінкою МНК тоді і тільки тоді, коли значення дисперсій відхилень задовільняють певній системі нелінійних рівнянь. При цих умовах оцінки Ейткена та MHK параметрів $b$ та $c$ не будуть збігатися. Розглянуто застосування теореми для деяких випадків конкретної кількості точок спостереження та однакових значеннях дисперсій у вузлах, симетричних відносно точки $\frac{1}{2}$.

\section{ЛIтерАТУРА}

1. Anderson T. The statistical analysis of time series. Moscow: Mir, 1976. 756 p. (in Russian)

2. Demidenko E. Z. Linear and nonlinear regression. Moscow: Finance and Statistics, 1981. 304 p. (in Russian)

3. Grenander Ulf On the estimation of the regression coefficients in the case of the autocorrelated disturbance. Ann. Math. Statist. 1954. 25. P. 252-272.

4. Zyskind G. On cannonical forms, non-negative covariance matrices, and best and simple least squares linear estimators in linear models. Ann. Math. Statist. 1967. 38. P. 1092-1109.

5. Magness T. A., McGuire J. B. Comparison of least squares and minimum variance estimates of regression parameters. Ann. Math. Statist. 1962. 33. P. 462-470.

6. Rosenblatt M. Serial correlation in Some regression problems in the time series analysis. Proc. Third Berkelay Symposium on Mathematical Statistics and Probability (J. Neyman, ed.), Vol. 1, Univ. of California Press, Berkelay and Los Angeles. 1956. P. 165-186.

7. Watson G. S. Serial correlation in regression analysis, I. Biometrika. 1955. 42. P. 327-341.

8. Watson G. S. Linear least squares regression. Ann. Math. Statist. 1967. 38. P. 1679-1699.

9. Watson G. S., Hannan E. J. Serial correlation in regression analysis, II. Biometrika. 1956. 43. P. 436-449.

10. Belov A. G. About coincidence of the LS and Aitken estimations. Applied mathematics and Informatics. Proceedings of the faculty of the VMK Moscow State University. 2016. Vol. 51. P. 95-99. (in Russian)

11. Savkina M. Conditions for the coincidence of the IS and Aitken estimations of the parameters of the linear regression model. Journal of Numerical and Applied Mathematics. 2018. No. 3 (129). P. 36-44. (in Ukrainian)

Надійшла: 02.12.2019 / Прийнята: 20.12.2019 


\title{
УСЛОВИЯ СОВПАДЕНИЯ ОЦЕНОК МНК И ЭЙТКЕНА СТАРШЕГО КОЭФФИЦИЕНТА МОДЕЛИ КВАДРАТИЧНОЙ РЕГРЕССИИ
}

\author{
М. Ю. САВКИНА
}

Институт математики НАНУ , Киев, Украина, E-mail: marta@imath.kiev.ua

АннотАция. В работе в случае гетероскедастических независимых отклонений изучается регрессионная модель, функция которой имеет вид $f(x)=a x^{2}+b x+c$, где $a, b$ и $c$ - неизвестные параметры. Приближенные значения (наблюдения) функции $f(x)$ регистрируются в равноудаленных точках отрезка $[0,1]$. Доказанная в работе теорема утверждает, что оценка Эйткена старшего коэффициента квадратичной модели в случае нечетного количества точек наблюдения совпадает с его оценкой МНК тогда и только тогда, когда значение дисперсий отклонений удовлетворяют определенной системе нелинейных уравнений. При этих условиях оценки Эйткена и МНК параметров $b$ и $c$ не будут совпадать. Рассмотрено применение теоремы для некоторых случаев конкретного количества точек наблюдения и одинаковых значениях дисперсий в узлах, симметричных относительно точки $\frac{1}{2}$. Во всех этих случаях получено, что оценка MHК будет совпадать с оценкой Эйткена, если дисперсия в двух точках принимает произвольные значения, а во всех других - определенные значения, выражающееся через значения дисперсий в этих двух точках. КЛЮчЕВЫЕ СЛовА: метод наименьших квадратов, регрессионная модель, оценка Эйткена. 